Doi: $10.24234 /$ se.2020.2.2.230

\title{
MODELING OF SPEECH THERAPY ASSESSMENT IN REGIONAL PEDAGOGICAL AND PSYCHOLOGICAL SUPPORT CENTERS
}

\author{
AUTHORS' DATA: \\ Anna Aslanyan, Speech Therapist \\ Mary Izmirlyan Medical Centre, Armenia \\ Contacts: aslanyan.an@mail.ru
}

Gohar Hovyan, $\mathrm{PhD}$ in Education, Associated professor

Chair of Speech and Rehabilitative Therapy, Khachatur Abovyan Armenian State Pedagogical University, Armenia

University lecturer

Contacts: gohar.hovyan@mail.ru

\begin{abstract}
Scientific indication chains with practical knowledge to prepare professionals to work effectively with children with speech disorders and their families. The importance of scientific evidence from around the world on speech disorders, assessment, analysis, diagnosis and intervention and its implication with practical knowledge to prepare speech therapists to work with children and their families is obvious and crucial.
\end{abstract}

The significance of this issue becomes more valuable when changes and reforms are taking place within the educational system in the country - something that has happened in Armenia and still requires research and adjustments in the field. The following study is guided by the contemporary framework that directs the successful assessment of speech disorders in children: World Helth Organization's International Classification of Functioning Disability and Health Children and Youth (ICF-CY) (2007).

The article provides comprehensive, legislative, practical and clinical information for modeling speech therapy assessment within the scope of new reforms taken place in the country. 
Key words: speech therapy, assessment, children with speech disorders, International classification of function, support center, pedagogical and psychological support.

\section{INTRODUCTION}

Children with disabilities or children with special educational needs and their families are among the most vulnerable groups in the Armenian society (UNICEF, 2012). They can be in special schools, inclusive schools, orphanages or completely out of education, and very often don't have access to community based rehabilitation and early intervention services, having limited participation in different outdoor, art or cultural activities. According to the United Nations Convention of the Rights of Persons with Disabilities (2006), the Armenian government has ratified the commitment to "ensure and promote the full realization of all human rights and fundamental freedom for all persons with disabilities" (Article 1).

Like any reforms, educational reforms taken place in each period, unquestionably, cannot be supposed to be the reform of organization and implementation of education, as the implementation of such a comprehensive reform first of all implies adoption of relevant educational policies by upholding certain problematic issues in addition to the positive changes, cause certain problematic issues. All these issues certainly need to be examined, because the innovations in the educational field refer not only to the school learners, the teachers and parents but also penetrate into the life of the entire society and become the most effective means to combat discriminatory attitude; they develop and create favorable conditions and build an inclusive community to ensure the educational process of all learners (Harutyunyan, Hovyan, Saratikyan, Azatyan, Tanajyan \& Muradyan, 2019 p.4).

\section{REVIEW OF THE LITERATURE}

In Armenia, like in many countries of the world, there are a lot of data gaps regarding persons with disabilities, hindering the possibility to make informed policy choices in fulfilling their rights (Harutyunyan \& Hovyan, 2013). Only first steps toward including these persons into society, such as changing education policy and accepting inclusive education for children with 
special needs are done by different non-state organizations and governmental structures. Success with inclusion of children with special educational needs often depends on the perspectives of the teachers implementing the inclusion and specialists (speech therapist, special educators, psychologists, occupational therapists, social workers) working with these children and their families (Harutyunyan, Hovyan, Saratikyan, Azatyan, Tanajyan \& Muradyan, 2019 p.6).

Speech disorders are quite frequent in early childhood, the most common type of which is a phonological impairment (Dodd, 2014). Children with speech disorders might have difficulty learning writing, reading, the whole phonological system of the ambient language, which results in pattern-based speech errors affecting entire classes or features of sounds. These children face an increased risk of literacy difficulties, poor educational outcomes and low self-esteem compared to their typically developing peers (McCormack, Harrison, McLeod, \& McAllister, 2011). From this perspective, early speech therapy intervention is very much required for this group of children in school.

Only through broad assessment and analysis of the needs of children with special educational needs (conducted by different specialists) it is possible to realize and ensure the involvement of each child in the educational process to develop their communication, integration and learning abilities (McLeod \& Baker, 2017).

At the same time, according to Protocol decision of the Government of Armenia as of 18.02.2016 the plan and timetable of implementation of universal inclusive education in Republic of Armenia was regulated. According to this decision, the following structural changes have been done in all Armenian regions since 2016 to 2022:

- regional pedagogical-psychological support centers (through transferring special schools into support centers) are founded;

- staff of new formulated regional pedagogical-psychological support centers is trained;

- children from special schools are allocated to inclusive schools;

- $\quad$ raised scale of funding for children with special educational needs in accordance with child needs is implemented, etc.

Guided by Law of the Republic of Armenia on General Education (2014) (Article 17 Part 7 "The procedure for providing pedagogical-psychological support services for the organization 
of education") the pedagogical-psychological support services for the organization of student education is provided at three levels:

1) school level;

2) regional level;

3) republican level.

School-level services are provided to learners who have been evaluated and recognized as being in need of special education. School level services are provided by service of pedagogical and psychological assistance of general education institution (teacher's assistant, special educator, psychologist, social pedagogue and nurse).

Regional services are provided by the regional center of pedagogical and psychological support based on the application of the directorate of the general education institution or the child's legal representative. Regional center specialists visit schools and provide support to the school's assistant team for developing student support services. In case of a child who is not attending a school/kindergarten, the assistance is provided in the regional pedagogical and psychological support center (Order N 370-N of the Minister of Education and Science of the Republic of Armenia of April 13, 2017).

After the approval of the student's individual learning plan, the regional support center and school directors jointly sign and validate the load, schedule of the services provided to the school (learner, teacher, parent) by territorial center and the attendance schedule of the territorial center's specialists. In addition, when designing the schedule, it is advisable to plan a specialist's visit to the school at least once a week. The schedule is attached to the IIP. The regional support center provides the necessary consulting and professional assistance on learners educational process to the learner's parent, the members of the school's pedagogical-psychological team and teachers to ensure permanent relationship between parents, specialists, and teachers in the form of mutual visits, telecommunication and online consultation (Order N 370-N of the Minister of Education and Science of the Republic of Armenia of April 13, 2017). If the analysis of the results of a child's school-level assessment indicates the probability of a child's any disorder, the institution, with the parent's consent, applies to the regional support center to carry out a regional assessment. Regional 
assesment can also be carried out directly on the parent's request. According to the order of the principal of the regional support center, a group of multidisciplinary team specialists implementing regional assessment is formed which comprises at least 3 specialists of the regional center: a special educator, a speech therapist and a psychologist. This assessment lasts at least a week after which the child assessment report and conclusion is compiled and submitted (Harutyunyan, Hovyan, Saratikyan, Azatyan, Tanajyan \& Muradyan, 2019; Order N 370-N of the Minister of Education and Science of the Republic of Armenia of April 13, 2017).

At the same time Order N 370-N of the Minister of Education and Science of the Republic of Armenia of April 13, 2017 on approves the procedure for providing pedagogical-psychological support services for the organization of education and regulates the issues related to the provision of the above mentioned services. Revision and detailed analyses show that the procedure of child development peculiarities assessment need to be revised. The developmental difficulty assessment system is not free from insufficiencies. The scale of assessment provided by local governments to the specialists, where the child's developmental specificities are inserted and the need assessment is calculated, result in a distorted image, which, according to specialists, is often far from the real picture of child development and overcoming the difficulties (Harutyunyan, Hovyan, Saratikyan, Azatyan, Tanajyan \& Muradyan, 2019).

In order to implement the policy of universal inclusive education in the Republic of Armenia, in accordance with Article 17.1 of the Law of the Republic of Armenia on General Education, the criteria of pedagogical-psychological assessment of speech and special educational needs of children are currently applied to children were adopted and based on International classification of functioning, disability and health: children and youth developed by World Health Organization (WHO, ICF- CY, 2007). Despite the fact that WHO ICF based assessment might require more administrative resources, it still enables to have more comprehensive information about a child and to better understand his environment, which may promote or hinder his social inclusion, as well as to establish more targeted services for the rehabilitation and social inclusion of people with disabilities (Harutyunyan, 2017).

These criteria have been developed in accordance with the requirements of the Law of the Republic of Armenia on General Education. The current Law stipulates that in order to increase the accessibility, quality and efficiency of educational services, the educational institution provides 
pedagogical and psychological support services for the organization of education defined by the authorized state body (Article 12, point 3, part 7). It provides the application of effective measures for individual support to children with special educational needs in those places where the educational process is parallel with the reforms. At the same time, it helps to contribute to the educational and social development of children with special educational needs, including children with speech disorders and their inclusion in society. However, within the framework of these educational reforms, in terms of regional level assessment, certain problems arise in the process of speech therapy (diagnosis), as such an assessment process is currently based on five main functional disorders: voice and speech, hearing, vison, intellectual (mental) (Order N 370-N of the Minister of Education and Science of the Republic of Armenia of April 13, 2017, Form 4). From this perspective, it is possible that the classification based on dysfunction cannot be a basis for determining the type, period, duration and scope of pedagogical and psychological support services, as function assessment is itself a medical process that requires narrow professional intervention.

In order to determine the type and degree of speech disorder, as well as other questions related to the child's speech problems, the child first of all should be assessed by a speech therapist comprehensively (Levina, 2015). Within this frame, the following article has the aim to discuss the modeling of speech therapy assessment in regional pedagogical-psychological centers within the scope of recently taken reforms in Armenia. Examination of speech disorders is a key precondition for organizing speech therapy intervention and the effectiveness of speech therapy work depends on the proper organization of this process.

\section{METHOD}

In order to understand the state of the art of speech therapy assessment within the frame of regional pedagogical and psychological support centers in regards with policy and practical implementation changes and reforms, the existing legislative documents were investigated. Qualitative content analysis was conducted to reveal the spectrum of new legislative innovations and to describe lawmakers' evolving view of speech therapy assessment peculiarities. Qualitative content analysis is not a new approach to textual analysis. This method borrowed from the social 
and health sciences is used to analyze legal documents (Hall \& Wright, 2008). Thus, applying proven methods from the health sciences and communication studies can support large-scale analysis of legal transcripts (Hall \& Steiner, 2020).

This paper, based on the qualitative content analysis method, seeks to describe the approaches used to speech therapy assessment highlighted in the existing policy and its practical implication while organizing pedagogical and psychological support.

\section{DISCUSSION OF THE RESULTS}

According to the existing policy, the pedagogical-psychological assessment criteria for identification of the need for special education are used to organize the education of children with special educational needs in order to determine the increased amount of funding. According to the international and local legal acts regulating the education sector, inclusive education should be affordable, of a high quality and free; elementary and secondary education should be organized on an equal basis with other community members.

The importance of ensuring the necessary support for children with special educational needs in a well-established and well-defined full education system should have provided with a range of pedagogical conditions to ensure effective learning and inclusion in the educational process, which still remains unresolved (Harutyunyan, Hovyan, Saratikyan, Azatyan, Tanajyan \& Muradyan, 2019 p.10). Yet, according to N 6 Protocol Decision of RA Government on 18.02.2016 on the approval of Action Plan and schedule of introduction to Full Inclusive Education system, the number of pedagogical and psychological assistance services in schools implementing inclusive education has been reduced by the average annual number of students in need of special education. This is a serious obstacle for a child with physical and mental development problems to remain out of education system, as most of them, in fact, can't use pedagogical and psychological, rehabilitation and early interference services and has limited participation in community life. Thus, a key precondition for efficient assessment based on the WHO ICF-CY approach is the implementing specialists' objective, compassionate and professional attitude. Each specialist must bear responsibility for objective assessment and provided information (Harutyunyan, 2017b). 
Existing scientific studies aimed at organizing speech assessment according to the peculiarities of functional assessment are not completely sufficient for the diagnosis of speech disorders in Armenian-speaking children, and then for the organization of speech therapy intervention planned on the basis of that assessment (Alekseyeva, 2017; McLeod \& Baker, 2017; Walther-Thomas, Korinek, McLaughlin \& Williams, 2000). It means that this field requires multilevel and large-scale research. The need for the above-mentioned studies is urgent in the sense for the following reason: the model used in the speech therapy assessment process in regional pedagogical-psychological centers is going to be scientifically substantiated in Armenia for the first time. The new model of speech therapy assessment, based on WHO ICF-CY (2007) will provide an opportunity for functional assessment of the speech disorders on the one hand; on the other hand, to conduct intervention in order to overcome the speech problems of children with speech disorders in educational settings. Thus, WHO ICF-CY as a model provides a conceptual framework for a range of information that can be used to provide educational, social and rehabilitative care to a person, including assessment, intervention, prevention, health promotion, increased participation, elimination or reduction of barriers and promotion of public support, as well as the provision of supporting factors (Harutyunyan, 2017a).

In order to solve the problem, we consider it necessary first to analyze the current system of education of children with special educational needs in order to determine the increased amount of funding and then apply the criteria of pedagogical-psychological assessment of the need for the children who require special education (Order N 370-N of the Minister of Education and Science of the Republic of Armenia of April 13, 2017).

In order to provide speech therapy support at the proper level, professionals in the field must first be able to assess the child's speech problems according to the general criteria based on the classification of speech disorders accepted in modern speech therapy. Unfortunately, in most cases, the above regularities of evaluation are not taken into account in regional pedagogical and psychological support centers. In this regards, while assessing the legislative documents and getting acquainted with the practical work of speech therapists from support centers, the following issues become more than urgent.

First of all, it is necessary to conduct review and analyses of existing dysfunctions and reflect to the type, period, duration and content of pedagogical and psychological support. At the 
same time review of filed related modern scientific literature is highly required. Exploring the work of speech therapists working in pedagogical and psychological support centers in the process of speech therapy assessment has very significant value. Understanding of targeted support methods not only in pedagogical and psychological support centers, but also in school while learning process is quite informative in regards of organization speech therapy assessment and future intervention. Based on the existing policies, it is essential to unify cooperative-consulting activities for the effective organization of the educational process of a child with a speech disorder within the framework of the services provided to teachers, in order to develop more favorable, effective ways of working with such children. And finally, the review of existing speech assessment tools, anamnestic data, and speech assessment cards is also important.

The importance of modeling speech therapy assessment in the current education system has been identified and substantiated by us. New model based on WHO ICF-CY (2007) is developed. This model will offer possibilities for an effective speech therapy assessment through a detailed methodological guidance which will enhance speech therapy assessment process in pedagogical and psychological support centers.

The functional assessment new model developed through the WHO ICF-CY (2007) must be aimed at the study and analysis of children's speech, voice, temporal rhythmic structure, general speech impairment, alalia, aphasia, oral and written language developmental levels, compliance with native language assessments. Degree of skills according to the relevant qualifications are described as light, moderate, severe and profound.

\section{RECOMMENDATION}

Review of the existing policies and qualitative content analysis that was conducted to reveal the spectrum of new legislative innovations in the field of speech therapy assessment provided by regional pedagogical and psychological support centers allowed us to come up with some concrete suggestions. First of all, it is proposed to elaborate methodological guidance 'Speech disorders' assessment and implementation of speech therapy intervention in support centers" which will be developed by our research team precisely for speech therapists working in pedagogical and psychological support centers and speech therapists working in inclusive schools. This 
methodological guidance will help to improve work performance of speech therapists, increase the efficiency of inclusive education within the framework of educational reforms, as well as choose the main ways of collaborative teamwork with children with special educational needs and their families, and indicate even more effective ways of organizing pedagogical and psychological work within the frame of inclusiveness.

Helping speech therapist to overcome their daily difficulties connected with assessment and speech therapy intervention we aim to acquaint them with the increase of professional knowledge and learning new practical skills of pedagogical-psychological assessment in terms of innovations in the field, and mastery of modern approaches.

Summarizing the invaluable role of speech function in the process of child development and analyzing a number of patterns related to developmental problems: speech, level of mental and personal development, which are not considered only in the context of teaching and upbringing, it is important to mention that speech therapists should be as professional as possible in carrying out the complex process of assessing speech disorders while working with children with special educational needs. Every specialist should be able to create peaceful, safe, trusting atmosphere, make the assessment process more interesting, fun, enjoyable, provide appropriate support, promoting the teaching of alternative methods of communication to the children as individually as well as in the group. Only using these approaches, it is possible to organize and coordinate the learning process of students with speech disorders, determining the positive outcome of psychological, pedagogical and rehabilitative intervention.

As the activities of the multidisciplinary team in the pedagogical and psychological support centers are mostly aimed at supporting not only children, but teachers from the given institution, it is very important to collaborate closely with them and provide necessary consultation regarding the issues they rise.

\section{CONCLUSION}

Based on the recommendation provided above, the following conclusion might be expected with its findings: 
- Speech disorder assessment based on the criteria suggested by WHO ICF-CY (2007) allow to justify real existing problems that child is experiencing and find better ways of therapeutic intervention;

- Existence of methodological guidance allows to elaborate a new model of theoretically and scientifically based speech assessment system which will enable the implementation of effective speech therapy assessment in regional pedagogical and psychological support centers;

- The modeling of speech therapy in regional pedagogical-psychological support centers should be aimed at covering the general theoretical issues of speech therapy, classification of speech disorders, content of speech therapy assessment and methods of implementation, development of practical skills for organization of pedagogical and psychological assessment, as well as organization of pedagogical and psychological activities with children with special educational needs.

- The practical significance of the new assessment model will be determined by the means and methods of organizing speech therapy intervention, the complete development of children's speech and effective social adaptation.

Taking into consideration the fact that the research was carried out on the basis of the analysis of the existing legislation and problems, omissions, professional needs in the practical field of speech therapy, we believe that the modeling of speech therapy assessment is very timely in the sense that the existing reforms in the field of education in Armenia, connected with the shift to full inclusion, require a sufficient level of professional knowledge and skills, which will allow to improve professional activity and perform accordingly.

Regarding the availability of professional theoretical and practical materials proposed within the framework of pedagogical and psychological support, we believe, that the revised, supplemented training materials and analyzed results of outlined criteria will make the practical direction of the evaluation process effective. At the same time, it will enable the employees of the field to improve their professional activity with rich content-oriented materials.

Only after this implication will it be possible to reflect the weak points of existing legislation in the field and require purposeful actual changes. 


\section{REFERENCE LIST}

1. Alekseeva, G., S. (2017). Kriterii diagnosticheskoy ocenki logopedicheskikh problem u detey starshego doshkolnogo vozrasta dlya vibora optimalnikh korrekcionnikh zdorovyesberegayushikh tekhnologiy, Nauchno-metodicheskiy elektronniy journal Koncept, N52

2. Dodd, B. (2014). Differential diagnosis of pediatric speech sound disorder. Current Developmental Disorders Reports, 1(3), 189-196.

3. Hall, M., D., \& Steiner, R. (2020). Policy content analysis: Qualitative method for analyzing sub-national insect pollinator legislation, MethodsX, Volume 7

4. Hall, M., A., \& Wright, R., F. (2008). Systematic content analysis of judicial opinions, Calif. Law Rev., 96, p. 63

5. Harutyunyan M., R. (2017a). Discussion of required reforms in the field of disability in Armenia and the first steps of their implementation, Proceedings of the International Scientific Conference, Science of the XXI century: problems and prospects of researches Vol.3

6. Harutyunyan M., R. (2017b). WHO International Classification of function as a disability determination model in Armenia, International Academy Journal Web of Scholar 5 (14).

7. Harutyunyan, M., Hovyan, G., Saratikyan, L., Azatyan, T., Tanajyan, K., \& Muradyan, S. (2019). Evaluation of universal inclusion processes consistence in Tavush, Lori and Syunik regions in the Republic of Armenia, Report conducted by Khachatur Abovyan Armenian State Pedagogical University Research center on inclusive community and disability issues, Caritas Armenian and Caritas Germany

8. Harutyunyan, Z., V., \& Hovyan, G., R. (2013). Bazmamasnagitakan timayin ashkhatanqy vorpes krtutyan ardyunavet kazmakerpman nakhapayman, Mankavarjutyun gitamethodakan amsagir, 7, ej 21-25

9. Law of the Republic of Armenia on General Education. 2014.

10. Levina, R., E. (2015). Rech i razvitie anomalnikh detey, Almanakh instituta korrekcionnoy pedagogiki RAO. 22-1 (22). s. 107-128.

11. McCormack, J., Harrison, L. J., McLeod, S., \& McAllister, L. (2011). A nationally representative study of the association between communication impairment at 4-5 years 
and children's life activities at 7-9 years. Journal of Speech, Language, and Hearing Research, 54(5), 1328-1348

12. McLeod, S., \& Baker, E. (2017). Children's Speech: An Evidence-Based Approach to Assessment and Intervention. U.S.: Pearson.

13. Order N 370-N of the Minister of Education and Science of the Republic of Armenia of April 13, 2017 on approving the procedure for providing pedagogical-psychological support services for the organization of education

14. RA Government Session 18.02.2016 Protocol Decision No. 6 on approving the action plan and schedule for the implementation of the universal inclusive education system.

15. Walther-Thomas, Ch., Korinek, L., McLaughlin, V., L., Williams, B., T. (2000). Collaboration for Inclussive Education: Developing Successful Programs. Boston London - Toronto: Allyn \& Bacon.

16. World Health Organization (2007). International classification of functioning, disability and health: children \& youth version: ICF-CY.

17. UNICEF (2012). Humanitarian Action for children, doi: http://www.unicef.org/hac2012

18. United Nations Convention on the Rights of Persons with Disabilities and Optional Protocol. 2006. 